\title{
AN ERROR ESTIMATE FOR TWO-DIMENSIONAL STOKES DRIVEN CAVITY FLOW
}

\author{
ZHIQIANG CAI AND YANQIU WANG
}

\begin{abstract}
Discontinuous velocity boundary data for the lid driven cavity flow has long been causing difficulties in both theoretical analysis and numerical simulations. In finite element methods, the variational form for the driven cavity flow is not valid since the velocity is not in $\boldsymbol{H}^{1}$. Hence standard error estimates do not work. By using only $\mathbf{W}^{1, r}(1<r<2)$ regularity and constructing a continuous approximation to the boundary data, here we present error estimates for both the velocity-pressure formulation and the pseudostressvelocity formulation of the two-dimensional Stokes driven cavity flow.
\end{abstract}

\section{INTRODUCTION}

The purpose of this paper is to provide strict error estimates for different finite element approximations of the two-dimensional Stokes lid driven cavity flow. The two-dimensional Stokes driven cavity problem has been thoroughly studied in numerous references. The main difficulty of this problem comes from the discontinuity of the velocity boundary data at corners. Although the structure of its solution is well-understood through the description of corner eddies and the use of bi-orthogonal series 20,21, 23, there are still many things that need to be clarified concerning its finite element approximation. Due to the discontinuous boundary data, the velocity is not in $\boldsymbol{H}^{1}$. Hence the usual variational form for Stokes equations is no longer valid for the driven cavity problem, which will be further explained in Section 3. Although in practice, many tend to ignore this and apply the discontinuous boundary data directly to the finite element discretization. In this paper, we attempt to give a strict mathematical explanation that will bridge the gap between theory and practice. To deal with this situation, our main approach is to construct a continuous approximation to the discontinuous boundary data. Fractional Sobolev norms are used in order to derive error estimates. Two different finite element formulations, the velocity-pressure formulation and the pseudostressvelocity formulation, will be considered. Their error estimates, the main result of this paper, will be given in Section 3 .

We first introduce some notation. Let $\Omega$ be a convex polygon. Denote $\mathbb{R}^{2}$ to be the field of two-dimensional vector functions and $\mathbb{M}_{2}$ to be the field of $2 \times 2$ matrix functions. Throughout the paper, we adopt the convention that a Greek character denotes a $2 \times 2$ matrix and a bold Latin character in lowercase denotes a vector.

Received by the editor September 13, 2007 and in revised form, May 7, 2008.

2000 Mathematics Subject Classification. Primary 65N15, 65N30, 76D07.

(C)2008 American Mathematical Society

Reverts to public domain 28 years from publication 
Let $\boldsymbol{\tau}=\left(\tau_{i j}\right)_{1 \leq i, j \leq 2} \in \mathbb{M}_{2}$ and $\boldsymbol{v}=\left(v_{1}, v_{2}\right)^{t} \in \mathbb{R}^{2}$, define

$$
\operatorname{div} \boldsymbol{v}=\frac{\partial v_{1}}{\partial x}+\frac{\partial v_{2}}{\partial y} \quad \text { and } \quad \operatorname{div} \boldsymbol{\tau}=\left(\begin{array}{l}
\frac{\partial \tau_{11}}{\partial x}+\frac{\partial \tau_{12}}{\partial y} \\
\frac{\partial \tau_{21}}{\partial x}+\frac{\partial \tau_{22}}{\partial y}
\end{array}\right) .
$$

In the above definitions, all derivatives are taken in the weak sense. Define the inner-products between vectors and between $2 \times 2$ matrices, respectively, by

$$
\boldsymbol{u} \cdot \boldsymbol{v}=u_{1} v_{1}+u_{2} v_{2} \quad \text { and } \quad \boldsymbol{\sigma}: \boldsymbol{\tau}=\sum_{1 \leq i, j \leq 2} \sigma_{i j} \tau_{i j} .
$$

Let $L^{2}(\Omega)$ be the set of square integrable functions on $\Omega$ and $W^{s, r}(\Omega)$, where $s$ is a real number and $1 \leq r \leq \infty$, be the Sobolev space 9 , 17] on $\Omega$. Let $C_{0}^{\infty}(\Omega)$ be the space of infinitely differentiable functions with compact support in $\Omega$. Denote $W_{0}^{s, r}(\Omega)$ to be the closure of $C_{0}^{\infty}(\Omega)$ under the $W^{s, r}(\Omega)$ norm. When $r=2$, both $W^{s, r}(\Omega)$ and $W_{0}^{s, r}(\Omega)$ are Hilbert spaces and are usually denoted by $H^{s}(\Omega)$ and $H_{0}^{s}(\Omega)$. Finally, the fractional Sobolev norm on $\partial \Omega$ is defined as follows. Let $s=m+t$, where $m \geq 0$ is an integer and $0<t<1$. Let $1<r<\infty$. For $v \in W^{s, r}(\partial \Omega)$, define

$$
\|v\|_{s, r, \partial \Omega}=\left(\|v\|_{m, r, \partial \Omega}^{r}+\sum_{|\alpha|=m} \int_{\partial \Omega} \int_{\partial \Omega} \frac{\left|D^{\alpha} v(\boldsymbol{x})-D^{\alpha} v(\boldsymbol{y})\right|^{r}}{|\boldsymbol{x}-\boldsymbol{y}|^{1+t r}} d \boldsymbol{x} d \boldsymbol{y}\right)^{1 / r} .
$$

Remark 1.1. The trace theorem is more complicated on polygonal domains than on smooth domains [17. Let $\Gamma_{i}, i=1, \ldots, k$ be the edges of the polygonal domain $\Omega$. If $s-1 / r$ is not an integer, the trace of a function in $W^{s, r}(\Omega)$ lies in $\prod_{i=1}^{k} W^{s-1 / r, r}\left(\Gamma_{i}\right)$ and satisfies certain matching conditions at the corners of $\Omega$. For example, the trace of $W^{2, r}(\Omega), r>2$, is in $\prod_{i=1}^{k} W^{2-1 / r, r}\left(\Gamma_{i}\right)$ and must be continuous across the corners. However, this will not affect approximation orders discussed in the following sections. A similar situation and explanation can be found in [18. Therefore, for simplicity, we will use the unified notation $W^{s, r}(\partial \Omega)$ for traces instead of using $\prod_{i=1}^{k} W^{s, r}\left(\Gamma_{i}\right)$ with matching conditions.

It is natural to extend the above spaces to fields of vector functions and matrix functions by using product spaces. For example, $\mathbf{W}^{s, r}\left(\Omega, \mathbb{R}^{2}\right)$ and $\mathbf{W}^{s, r}\left(\Omega, \mathbb{M}_{2}\right)$ denote the Sobolev spaces over the field of vector functions and $2 \times 2$ matrix functions. Other notations, such as $\boldsymbol{H}^{s}\left(\Omega, \mathbb{R}^{2}\right), \boldsymbol{H}^{s}\left(\Omega, \mathbb{M}_{2}\right), \mathbf{L}^{2}\left(\Omega, \mathbb{R}^{2}\right)$ and $\mathbf{L}^{2}\left(\Omega, \mathbb{M}_{2}\right)$ are defined in the same fashion. For simplicity, denote $\|\cdot\|_{s, r, \Omega}$ and $|\cdot|_{s, r, \Omega}$ to be the Sobolev norm and semi-norm with indices $s$ and $r$ over scalar, vector or matrix function fields, depending on the type of the function. When $r=2$, we usually suppress $r$ in the subscript and denote the Sobolev norm and semi-norm by $\|\cdot\|_{s, \Omega}$ and $|\cdot|_{s, \Omega}$. Denote $(\cdot, \cdot)$ and $\langle\cdot, \cdot\rangle$ to be the $\mathbf{L}^{2}$ inner-product and the duality form, respectively, over scalar, vector or matrix function fields.

Define

$$
\boldsymbol{H}\left(\operatorname{div}, \Omega, \mathbb{M}_{2}\right)=\left\{\boldsymbol{\sigma} \in \mathbf{L}^{2}\left(\Omega, \mathbb{M}_{2}\right) \text { such that } \operatorname{div} \boldsymbol{\sigma} \in \mathbf{L}^{2}\left(\Omega, \mathbb{R}^{2}\right)\right\}
$$

with the norm

$$
\|\boldsymbol{\sigma}\|_{\boldsymbol{H}\left(\operatorname{div}, \Omega, \mathbb{M}_{2}\right)}^{2}=(\boldsymbol{\sigma}, \boldsymbol{\sigma})+(\operatorname{div} \boldsymbol{\sigma}, \operatorname{div} \boldsymbol{\sigma}) .
$$

Let $\boldsymbol{n}$ be the unit outward normal vector along $\partial \Omega$. By the trace theorem, we have $\left.\boldsymbol{\sigma n}\right|_{\partial \Omega} \in \boldsymbol{H}^{-1 / 2}\left(\partial \Omega, \mathbb{R}^{2}\right)$ for all $\boldsymbol{\sigma} \in \boldsymbol{H}\left(\operatorname{div}, \Omega, \mathbb{M}_{2}\right)[15$. 
In Section 2, we describe the velocity-pressure formulation and the pseudostressvelocity formulation for two-dimensional Stokes equations. Also given are the standard finite element approximation and error estimates for these two formulations. In Section 3, we discuss how to deal with the discontinuous velocity boundary condition of the driven cavity problem. Detailed error estimates are given. Finally, some technical inequalities used in our analysis will be proved in Appendices A and B.

\section{Stokes equations}

Consider the two-dimensional steady-state Stokes equations:

$$
\begin{cases}-\Delta \boldsymbol{u}+\nabla p=\boldsymbol{f} & \text { in } \Omega, \\ \operatorname{div} \boldsymbol{u}=0 & \text { in } \Omega, \\ \boldsymbol{u}=\boldsymbol{g} & \text { on } \partial \Omega,\end{cases}
$$

where $\boldsymbol{u}$ is the velocity, $p$ is the pressure, $\boldsymbol{f}$ is the external force and $\boldsymbol{g}$ is the velocity boundary data satisfying $\int_{\partial \Omega} \boldsymbol{g} \cdot \boldsymbol{n} d s=0$. The Stokes equations usually arise either from the approximation of a low-Reynolds-number flow or from the time discretization of Navier-Stokes equations.

The Stokes equations have the following existence and regularity property [15, 16.

Theorem 2.1. Let $\Omega$ be a convex polygon. Given $\boldsymbol{f} \in \mathbf{W}^{m, r}\left(\Omega, \mathbb{R}^{2}\right)$ and $\boldsymbol{g} \in$ $\mathbf{W}^{m+2-1 / r, r}\left(\partial \Omega, \mathbb{R}^{2}\right)$ where $m=-1,0$ and $1<r \leq 2$. Then Problem (2.1) has a unique solution $(\boldsymbol{u}, p) \in \mathbf{W}^{m+2, r}\left(\Omega, \mathbb{R}^{2}\right) \times W^{m+1, r}(\Omega) / \mathbb{R}$ which satisfies

$$
\|\boldsymbol{u}\|_{m+2, r, \Omega}+\|p\|_{m+1, r, \Omega} \leq C_{r}\left(\|\boldsymbol{f}\|_{m, r, \Omega}+\|\boldsymbol{g}\|_{m+2-1 / r, r, \partial \Omega}\right),
$$

where $C_{r}$ is a positive constant independent of $\boldsymbol{f}$ and $\boldsymbol{g}$ but may depend on $r$.

Remark 2.2. In both two-dimension and three-dimension, similar existence and regularity results for $m=-1,0$ and $1<r<\infty$ are well-known under assumptions $\partial \Omega \in C^{2}$ or $\partial \Omega \in C^{1,1}$ (see [1, 8]). For Lipschitz continuous boundary $\partial \Omega \in C^{0,1}$, it was shown in 14] that the result of Theorem 2.1 holds for $m=-1$ and $1<r<\infty$ if the Lipschitz constant of the boundary $\partial \Omega$ is smaller than a constant depending only on the dimension and $r$. For convex polygons, the result of Theorem 2.1] was proved in [16], which was also especially pointed out in Remark 5.6 of [15].

In the following subsections, two different variational formulations and corresponding finite element approximations for problem (2.1) will be given. We always assume that $\Omega$ is a convex polygon.

2.1. The velocity-pressure formulation and its discretization. Assume that $\boldsymbol{f} \in \boldsymbol{H}^{-1}\left(\Omega, \mathbb{R}^{2}\right)$ and $\boldsymbol{g} \in \boldsymbol{H}^{1 / 2}\left(\partial \Omega, \mathbb{R}^{2}\right)$. Then the velocity-pressure formulation for problem (2.1) is: Find $\boldsymbol{u} \in \boldsymbol{H}^{1}\left(\Omega, \mathbb{R}^{2}\right),\left.\boldsymbol{u}\right|_{\partial \Omega}=\boldsymbol{g}$ and $p \in L^{2}(\Omega) / \mathbb{R}$ such that

$$
\begin{cases}(\nabla \boldsymbol{u}, \nabla \boldsymbol{v})-(\operatorname{div} \boldsymbol{v}, p)=\langle\boldsymbol{f}, \boldsymbol{v}\rangle & \text { for all } \boldsymbol{v} \in \boldsymbol{H}_{0}^{1}\left(\Omega, \mathbb{R}^{2}\right), \\ (\operatorname{div} \boldsymbol{u}, q)=0 & \text { for all } q \in L^{2}(\Omega) / \mathbb{R} .\end{cases}
$$

Problem (2.2) is a saddle-point problem and has been extensively studied for decades [4, 11, 15. To discretize this problem, several different finite element spaces have been proposed. They can be divided into two major groups according to whether the discrete inf-sup condition (LBB condition) is satisfied or not. If the 
LBB condition is satisfied, the pair of finite element spaces is called stable. Otherwise it is called unstable and requires a special stabilization process. For simplicity, only stable finite element pairs will be considered here. We will investigate the $P_{2}-P_{1}$ Taylor-Hood element [19] and the $P_{2}^{+}-P_{-1}$ conforming Crouzeix-Raviart element [10. The $P_{2}-P_{1}$ Taylor-Hood element uses $P_{2}$ piecewise continuous polynomials to approximate the velocity and $P_{1}$ piecewise continuous polynomials to approximate the pressure. The $P_{2}^{+}-P_{-1}$ conforming Crouzeix-Raviart element uses $P_{2}$ piecewise continuous polynomials plus bubble functions to approximate the velocity and discontinuous piecewise $P_{1}$ polynomials to approximate the pressure. Details of these elements are skipped since they can be found from numerous sources.

Let $\mathcal{T}_{h}$ be a quasi-uniform triangulation of $\Omega$ with characteristic mesh size $h$. For simplicity, in the rest of this paper, $\lesssim$ is used to denote "less than or equal to" with a factor $c$ independent of the mesh size $h$ or other parameters appearing in the inequality. Assume $\boldsymbol{f} \in \mathbf{L}^{2}\left(\Omega, \mathbb{R}^{2}\right)$ and $\boldsymbol{g} \in \boldsymbol{H}^{3 / 2}\left(\partial \Omega, \mathbb{R}^{2}\right)$. Let $\left(\mathbf{U}_{h}, Q_{h}\right) \subset$ $\left(\boldsymbol{H}^{1}\left(\Omega, \mathbb{R}^{2}\right), L^{2}(\Omega) / \mathbb{R}\right)$ be the $P_{2}-P_{1}$ or the $P_{2}^{+}-P_{-1}$ finite element spaces defined over $\mathcal{T}_{h}$, with the boundary condition $\left.\boldsymbol{u}_{h}\right|_{\partial \Omega}=\boldsymbol{g}_{h}$ for $\boldsymbol{u}_{h} \in \mathbf{U}_{h}$. Here $\left.\boldsymbol{g}_{h} \in\left(\mathbf{U}_{h}\right)\right|_{\partial \Omega}$ can be either the $\boldsymbol{H}^{3 / 2}$ projection or the nodal value interpolation of $\boldsymbol{g}$, where the $\boldsymbol{H}^{3 / 2}$ projection is defined by

$$
\left\|\boldsymbol{g}-\boldsymbol{g}_{h}\right\|_{3 / 2, \partial \Omega}=\min _{\left.\boldsymbol{\chi} \in\left(\mathbf{U}_{h}\right)\right|_{\partial \Omega}}\|\boldsymbol{g}-\boldsymbol{\chi}\|_{3 / 2, \partial \Omega}
$$

Define the space $\stackrel{\circ}{\mathbf{U}}_{h} \subset \boldsymbol{H}_{0}^{1}\left(\Omega, \mathbb{R}^{2}\right)$ similar to $\mathbf{U}_{h}$ but with homogeneous boundary conditions. Consider the discrete formulation for problem (2.2): Find $\boldsymbol{u}_{h} \in \mathbf{U}_{h}$ and $p_{h} \in Q_{h}$ such that

$$
\begin{cases}\left(\nabla \boldsymbol{u}_{h}, \nabla \boldsymbol{v}_{h}\right)-\left(\operatorname{div} \boldsymbol{v}_{h}, p_{h}\right)=\left\langle\boldsymbol{f}, \boldsymbol{v}_{h}\right\rangle & \text { for all } \boldsymbol{v}_{h} \in \stackrel{\circ}{\mathbf{U}}_{h}, \\ \left(\operatorname{div} \boldsymbol{u}_{h}, q_{h}\right)=0 & \text { for all } q_{h} \in Q_{h} .\end{cases}
$$

The following error estimate is well known [11, 13, 15]:

Theorem 2.3. Assume $\boldsymbol{f} \in \mathbf{L}^{2}\left(\Omega, \mathbb{R}^{2}\right)$ and $\boldsymbol{g} \in \boldsymbol{H}^{3 / 2}\left(\partial \Omega, \mathbb{R}^{2}\right)$. Let $(\boldsymbol{u}, p)$ be the solution to problem (2.2) and let $\left(\boldsymbol{u}_{h}, p_{h}\right)$ be the solution to problem (2.3). Then

$$
\left|\boldsymbol{u}-\boldsymbol{u}_{h}\right|_{1, \Omega}+\left\|p-p_{h}\right\|_{0, \Omega} \lesssim h\left(\|\boldsymbol{u}\|_{2, \Omega}+\|p\|_{1, \Omega}\right) .
$$

Moreover, if $\boldsymbol{g}=\mathbf{0}$, then

$$
\left\|\boldsymbol{u}-\boldsymbol{u}_{h}\right\|_{0, \Omega} \lesssim h^{2}\left(\|\boldsymbol{u}\|_{2, \Omega}+\|p\|_{1, \Omega}\right)
$$

Remark 2.4. We gain one more order in the approximation if $\boldsymbol{u} \in \boldsymbol{H}^{3}\left(\Omega, \mathbb{R}^{2}\right)$ and $p \in H^{2}(\Omega)$. That is

$$
\left|\boldsymbol{u}-\boldsymbol{u}_{h}\right|_{1, \Omega}+\left\|p-p_{h}\right\|_{0, \Omega} \lesssim h^{2}\left(|\boldsymbol{u}|_{3, \Omega}+|p|_{2, \Omega}\right)
$$

However, when the essential boundary condition is non-homogeneous, analysis for the $\mathbf{L}^{2}$ error estimate of the velocity is much more complicated. This issue has been thoroughly discussed in [13, 18]. According to their results,

Theorem 2.5. Under the same assumptions as in Theorem 2.3 , the $\mathbf{L}^{2}$ error estimate of the velocity is:

- if $\boldsymbol{g}_{h}$ is the $\boldsymbol{H}^{3 / 2}$ projection of $\boldsymbol{g}$, then

$$
\left\|\boldsymbol{u}-\boldsymbol{u}_{h}\right\|_{0, \Omega} \lesssim h^{2}\left(\|\boldsymbol{u}\|_{2, \Omega}+\|p\|_{1, \Omega}+\|\boldsymbol{g}\|_{3 / 2, \partial \Omega}\right) ;
$$


- if $\boldsymbol{g}_{h}$ is the nodal value interpolation of $\boldsymbol{g}$ and $\boldsymbol{g} \in \boldsymbol{H}^{2}\left(\partial \Omega, \mathbb{R}^{2}\right)$, then

$$
\left\|\boldsymbol{u}-\boldsymbol{u}_{h}\right\|_{0, \Omega} \lesssim h^{2}\left(\|\boldsymbol{u}\|_{2, \Omega}+\|p\|_{1, \Omega}+\|\boldsymbol{g}\|_{2, \partial \Omega}\right) .
$$

Theoretically, the approximation using the nodal value interpolation on the boundary is not optimal for $\boldsymbol{g} \in \boldsymbol{H}^{3 / 2}\left(\partial \Omega, \mathbb{R}^{2}\right)$, although the authors of [13] have presented some numerical results suggesting that one may still get optimal convergence in practice. The other possibility, using the $\boldsymbol{H}^{3 / 2}$ projection, is impractical although it does give an optimal error estimate. It is mentioned here just for the theoretical purpose.

2.2. The pseudostress-velocity formulation and its discretization. In this subsection we describe the pseudostress-velocity formulation [6, 7] for Problem (2.1). Let $\mathcal{A}: \mathbb{M}_{2} \rightarrow \mathbb{M}_{2}$ be a fourth order tensor defined by $\mathcal{A} \boldsymbol{\tau}=\boldsymbol{\tau}-\left(\frac{1}{2} \operatorname{tr} \boldsymbol{\tau}\right) \mathbf{I}$, for all $\boldsymbol{\tau} \in \mathbb{M}_{2}$. Here $\operatorname{tr} \boldsymbol{\tau}$ is the trace of $\boldsymbol{\tau}$ and $\mathbf{I}$ is the $2 \times 2$ identity matrix. Notice that $\mathcal{A}$ is a projection onto the trace-free subspace of $\mathbb{M}_{2}$ and

$$
\operatorname{Ker}(\mathcal{A})=\{f \mathbf{I} \text { for all scalar functions } f\} .
$$

It is clear that $(\mathcal{A} \boldsymbol{\tau}, \boldsymbol{\tau}) \leq(\boldsymbol{\tau}, \boldsymbol{\tau})$ for all $\boldsymbol{\tau} \in \boldsymbol{H}\left(\mathbf{d i v}, \Omega, \mathbb{M}_{2}\right)$.

By introducing the pseudostress $\boldsymbol{\sigma}=-p \mathbf{I}+\nabla \boldsymbol{u}$, which is not necessarily symmetric, System (2.1) can be rewritten as:

$$
\begin{cases}\operatorname{div} \boldsymbol{\sigma}=-\boldsymbol{f} & \text { in } \Omega, \\ \mathcal{A} \boldsymbol{\sigma}-\nabla \boldsymbol{u}=\mathbf{0} & \text { in } \Omega, \\ \left.\boldsymbol{u}\right|_{\partial \Omega}=\boldsymbol{g} . & \end{cases}
$$

The incompressible constraint $\operatorname{div} \boldsymbol{u}=0$ is enforced through $\operatorname{div} \boldsymbol{u}=\operatorname{tr}(\nabla \boldsymbol{u})=$ 0 . Notice that $\operatorname{tr} \boldsymbol{\sigma}=-2 p$ is unique up to a constant related to the hydrostatic pressure. Therefore, it needs to satisfy the compatibility condition

$$
\int_{\Omega} \operatorname{tr} \boldsymbol{\sigma} d x=0
$$

Remark 2.6. The advantage of using the pseudostress instead of the symmetric physical stress $\tilde{\boldsymbol{\sigma}}=-p \mathbf{I}+\left(\nabla \boldsymbol{u}+(\nabla \boldsymbol{u})^{T}\right)$ in the formulation is that, the finite element discretization for the mixed system is greatly simplified [6, 7]. For comparison, one may also check the mixed finite element construction for the symmetric physical stress in [2, 4]. Finally, we point out that the stress $\tilde{\boldsymbol{\sigma}}$ and the pressure $p$ can be expressed algebraically in terms of the pseudostress $\boldsymbol{\sigma}$ by

$$
\tilde{\boldsymbol{\sigma}}=\boldsymbol{\sigma}+(\mathcal{A} \boldsymbol{\sigma})^{t}, \quad p=-\frac{1}{2} \operatorname{tr} \boldsymbol{\sigma} .
$$

Hence they can be computed in a post-processing procedure in the same accuracy as the approximation of $\boldsymbol{\sigma}$.

Define spaces

$$
\boldsymbol{\Sigma}=\boldsymbol{H}\left(\operatorname{div}, \Omega, \mathbb{M}_{2}\right) / \operatorname{span}\{\mathbf{I}\}=\left\{\boldsymbol{\tau} \in \boldsymbol{H}\left(\operatorname{div}, \Omega, \mathbb{M}_{2}\right) \text { such that } \int_{\Omega} \operatorname{tr} \boldsymbol{\tau} d x=0\right\}
$$

and $\mathbf{V}=\mathbf{L}^{2}\left(\Omega, \mathbb{R}^{2}\right)$. Then the variational form for equation (2.6) is: Given $\boldsymbol{f} \in$ $\mathbf{L}^{2}\left(\Omega, \mathbb{R}^{2}\right)$ and $\boldsymbol{g} \in \boldsymbol{H}^{1 / 2}\left(\partial \Omega, \mathbb{R}^{2}\right)$, find $\boldsymbol{\sigma} \in \boldsymbol{\Sigma}$ and $\boldsymbol{u} \in \mathbf{V}$ such that

$$
\begin{cases}(\mathcal{A} \boldsymbol{\sigma}, \boldsymbol{\tau})+(\operatorname{div} \boldsymbol{\tau}, \boldsymbol{u})=\langle\boldsymbol{g}, \boldsymbol{\tau} \boldsymbol{n}\rangle_{\partial \Omega}, & \text { for all } \boldsymbol{\tau} \in \boldsymbol{\Sigma} \\ (\operatorname{div} \boldsymbol{\sigma}, \boldsymbol{v})=-(\boldsymbol{f}, \boldsymbol{v}), & \text { for all } \boldsymbol{v} \in \mathbf{V}\end{cases}
$$


In the above mixed formulation, the velocity boundary condition becomes the natural boundary condition. Here $\langle\boldsymbol{g}, \boldsymbol{\tau} \boldsymbol{n}\rangle_{\partial \Omega}$ is well defined since $\boldsymbol{\tau} \boldsymbol{n} \in \boldsymbol{H}^{-1 / 2}\left(\partial \Omega, \mathbb{R}^{2}\right)$ for all $\boldsymbol{\tau} \in \boldsymbol{\Sigma}$.

According to [4], the existence and uniqueness of problem (2.7) follows from the well-known continuous inf-sup condition,

$$
\|\boldsymbol{v}\|_{0, \Omega} \lesssim \sup _{\boldsymbol{\tau} \in \boldsymbol{H}\left(\operatorname{div}, \Omega, \mathbb{M}_{2}\right)} \frac{(\operatorname{div} \boldsymbol{\tau}, \boldsymbol{v})}{\|\boldsymbol{\tau}\|_{\boldsymbol{H}\left(\operatorname{div}, \Omega, \mathbb{M}_{2}\right)}}, \quad \text { for all } \boldsymbol{v} \in \mathbf{V}
$$

and the following lemma [5]:

Lemma 2.7. For all $\boldsymbol{\tau} \in \boldsymbol{\Sigma}$, we have

$$
\|\boldsymbol{\tau}\|_{0, \Omega}^{2} \lesssim\left\|\mathcal{A}^{1 / 2} \boldsymbol{\tau}\right\|_{0, \Omega}^{2}+\|\operatorname{div} \boldsymbol{\tau}\|_{-1, \Omega}^{2}
$$

To discretize problem (2.7), a good finite element approximation to the space $\boldsymbol{\Sigma}$ is needed. A natural choice will be to use two copies of stable finite elements for the vector space $\boldsymbol{H}\left(\operatorname{div}, \mathbb{R}^{2}\right)$. There are several well-known stable mixed elements for the vector space $\boldsymbol{H}\left(\operatorname{div}, \mathbb{R}^{2}\right)$. Here we consider the lowest order Raviart-Thomas (RT) element $\left[22\right.$. On each triangle $T \in \mathcal{T}_{h}$, define $R T_{T}=\operatorname{span}\left\{\left(\begin{array}{l}1 \\ 0\end{array}\right),\left(\begin{array}{l}0 \\ 1\end{array}\right),\left(\begin{array}{l}x \\ y\end{array}\right)\right\}$. The degrees of freedom for the RT element are the zeroth order moments of the normal components on each edge of $T$. Define

$$
\boldsymbol{\Sigma}_{T}=\left\{\left(\begin{array}{ll}
\sigma_{11} & \sigma_{12} \\
\sigma_{21} & \sigma_{22}
\end{array}\right) \text { such that }\left(\sigma_{11}, \sigma_{12}\right) \in R T_{T} \text { and }\left(\sigma_{21}, \sigma_{22}\right) \in R T_{T}\right\} .
$$

Define the finite element space

$$
\boldsymbol{\Sigma}_{h}=\left\{\boldsymbol{\sigma} \in \boldsymbol{\Sigma} \text { such that }\left.\boldsymbol{\sigma}\right|_{T} \in \boldsymbol{\Sigma}_{T}\right\} .
$$

Notice that $\boldsymbol{\Sigma}_{h}$ inherits the constraint $\int_{\Omega} \operatorname{tr} \boldsymbol{\sigma} d x=0$ from the space $\boldsymbol{\Sigma}$.

Define the space $\mathbf{V}_{h} \subset \mathbf{L}^{2}\left(\Omega, \mathbb{R}^{2}\right)$ as follows:

$$
\mathbf{V}_{h}=\left\{\left(v_{1}, v_{2}\right)^{t} \text { such that } v_{1}, v_{2} \text { are constants on each } T \in \mathcal{T}_{h}\right\} .
$$

To show that $\left(\boldsymbol{\Sigma}_{h}, \mathbf{V}_{h}\right)$ form a stable pair of finite element spaces, one needs to prove the discrete inf-sup condition

$$
\left\|\boldsymbol{v}_{h}\right\|_{0, \Omega} \lesssim \sup _{\boldsymbol{\tau}_{h} \in \boldsymbol{\Sigma}_{h}} \frac{\left(\operatorname{div} \boldsymbol{\tau}_{h}, \boldsymbol{v}_{h}\right)}{\left\|\boldsymbol{\tau}_{h}\right\|_{\boldsymbol{H}\left(\operatorname{div}, \Omega, \mathbb{M}_{2}\right)}} \quad \text { for all } \boldsymbol{v}_{h} \in \mathbf{V}_{h}
$$

Actually, this follows easily from the facts that the RT element satisfies the discrete inf-sup condition [4] and that $\operatorname{span}\{\mathbf{I}\} \subset \operatorname{Ker}(\mathbf{d i v})$.

We also need to know whether $\boldsymbol{\Sigma}_{h}$, under the constraint $\int_{\Omega} \operatorname{tr} \boldsymbol{\sigma} d x=0$, is still a good approximation to $\boldsymbol{\Sigma}$. Indeed, denote $\mathbf{P}_{h}: \mathbf{L}^{2}\left(\Omega, \mathbb{R}^{2}\right) \rightarrow \mathbf{V}_{h}$ to be the $\mathbf{L}^{2}$ projection, then the following lemma holds [6].

Lemma 2.8. There exists a linear operator $\Pi_{h}: \boldsymbol{\Sigma} \cap \boldsymbol{H}^{1}\left(\Omega, \mathbb{M}_{2}\right) \rightarrow \boldsymbol{\Sigma}_{h}$ such that $\operatorname{div} \Pi_{h}=\mathbf{P}_{h} \operatorname{div}$ and, it holds for all $\boldsymbol{\sigma} \in \boldsymbol{\Sigma} \cap \boldsymbol{H}^{1}\left(\Omega, \mathbb{M}_{2}\right)$ that

$$
\left\|\boldsymbol{\sigma}-\Pi_{h} \boldsymbol{\sigma}\right\|_{0, \Omega} \lesssim h|\boldsymbol{\sigma}|_{1, \Omega}
$$

and

$$
\left\|\operatorname{div} \boldsymbol{\sigma}-\operatorname{div}\left(\Pi_{h} \sigma_{h}\right)\right\|_{0, \Omega} \lesssim|\operatorname{div} \boldsymbol{\sigma}|_{0, \Omega} .
$$

Furthermore, if $\operatorname{div} \boldsymbol{\sigma} \in \boldsymbol{H}^{1}\left(\Omega, \mathbb{R}^{2}\right)$, then

$$
\left\|\operatorname{div} \boldsymbol{\sigma}-\operatorname{div}\left(\Pi_{h} \boldsymbol{\sigma}_{h}\right)\right\|_{0, \Omega} \lesssim h|\operatorname{div} \boldsymbol{\sigma}|_{1, \Omega} .
$$


Finally, we give an error estimate for the mixed finite element approximation.

Theorem 2.9. Assume $\boldsymbol{f} \in \mathbf{L}^{2}\left(\Omega, \mathbb{R}^{2}\right)$ and $\boldsymbol{g} \in \boldsymbol{H}^{3 / 2}\left(\partial \Omega, \mathbb{R}^{2}\right)$. Let $(\boldsymbol{\sigma}, \boldsymbol{u})$ be the solution to problem (2.7) and let $\left(\boldsymbol{\sigma}_{h}, \boldsymbol{u}_{h}\right)$ be the solution to the discrete problem: Find $\boldsymbol{\sigma}_{h} \in \boldsymbol{\Sigma}_{h}$ and $\boldsymbol{u}_{h} \in \mathbf{V}_{h}$ such that

$$
\begin{cases}\left(\mathcal{A} \boldsymbol{\sigma}_{h}, \boldsymbol{\tau}_{h}\right)+\left(\operatorname{div} \boldsymbol{\tau}_{h}, \boldsymbol{u}_{h}\right)=\left\langle\boldsymbol{g}, \boldsymbol{\tau}_{h} \boldsymbol{n}\right\rangle_{\partial \Omega} & \text { for all } \boldsymbol{\tau}_{h} \in \boldsymbol{\Sigma}_{h} \\ \left(\boldsymbol{d i v} \boldsymbol{\sigma}_{h}, \boldsymbol{v}_{h}\right)=-\left(\boldsymbol{f}, \boldsymbol{v}_{h}\right) & \text { for all } \boldsymbol{v}_{h} \in \mathbf{V}_{h}\end{cases}
$$

Then

$$
\left\|\boldsymbol{\sigma}-\boldsymbol{\sigma}_{h}\right\|_{0, \Omega} \lesssim h|\boldsymbol{\sigma}|_{1, \Omega} \quad \text { and } \quad\left\|\boldsymbol{u}-\boldsymbol{u}_{h}\right\|_{0, \Omega} \lesssim h\left(|\boldsymbol{u}|_{1, \Omega}+|\boldsymbol{\sigma}|_{1, \Omega}\right)
$$

Furthermore, if $\operatorname{div} \boldsymbol{\sigma}=\operatorname{div} \boldsymbol{\sigma}_{h}$, then

$$
\left\|\boldsymbol{u}-\boldsymbol{u}_{h}\right\|_{0, \Omega} \lesssim h|\boldsymbol{u}|_{1, \Omega}+h^{2}|\boldsymbol{\sigma}|_{1, \Omega} .
$$

The proof of Theorem 2.9 follows directly from Theorem 2.1 Lemma 2.8, the stability of the RT element and the standard mixed finite element theory [4, 12. For the reader's convenience, the proof is given in Appendix A Also, the pressure $p$ can be approximated conveniently by $p_{h}=-\frac{1}{2} \operatorname{tr} \boldsymbol{\sigma}_{h}$ and

$$
\left\|p-p_{h}\right\|_{0, \Omega}=\left\|-\frac{1}{2} \operatorname{tr} \boldsymbol{\sigma}+\frac{1}{2} \operatorname{tr} \boldsymbol{\sigma}_{h}\right\|_{0, \Omega} \lesssim h|\boldsymbol{\sigma}|_{1, \Omega}
$$

\section{THE BOUNDARY CONDITION FOR LID DRIVEN CAVITY FLOW}

In this section, we study the discretization of the Stokes lid driven cavity flow using both the velocity-pressure and the pseudostress-velocity formulation. Consider the flow in a rectangular cavity generated by the uniform motion of the top lid. Let $\Omega=(0, a) \times(0, b)$, where $a$ and $b$ are positive numbers. The boundary of $\Omega$ is divided into two parts:

$$
\Gamma_{1}=\{x \in[0, a], y=b\} \quad \text { and } \quad \Gamma_{0}=(\partial \Omega) \backslash \Gamma_{1} .
$$

Define the velocity boundary condition by

$$
\left.\boldsymbol{u}\right|_{\partial \Omega}=\boldsymbol{g}^{d c}= \begin{cases}(1,0)^{t} & \text { on } \Gamma_{1}, \\ (0,0)^{t} & \text { on } \Gamma_{0} .\end{cases}
$$

By calculating the norm, one can see that $\boldsymbol{g}^{d c} \in \boldsymbol{H}^{1 / 2-\delta}\left(\partial \Omega, \mathbb{R}^{2}\right)$ for all $0<\delta<1 / 2$ but $\boldsymbol{g}^{d c} \notin \boldsymbol{H}^{1 / 2}\left(\partial \Omega, \mathbb{R}^{2}\right.$ ) (see [17]). Then by the Sobolev embedding theorem [17], it is clear that $\boldsymbol{g}^{d c} \in \mathbf{W}^{1-1 / r, r}\left(\partial \Omega, \mathbb{R}^{2}\right)$ for all $1<r<2$.

Consider the following driven cavity problem:

$$
\begin{cases}-\Delta \boldsymbol{u}+\nabla p=\mathbf{0} & \text { in } \Omega, \\ \operatorname{div} \boldsymbol{u}=0 & \text { in } \Omega, \\ \boldsymbol{u}=\boldsymbol{g}^{d c} & \text { on } \partial \Omega .\end{cases}
$$

By Theorem 2.1 problem (3.2) admits a unique solution

$$
(\boldsymbol{u}, p) \in\left(\mathbf{W}^{1, r}\left(\Omega, \mathbb{R}^{2}\right), W^{0, r}(\Omega) / \mathbb{R}\right) \quad \text { for } 1<r<2 .
$$

Recall that it requires at least $\boldsymbol{g} \in \boldsymbol{H}^{1 / 2}\left(\partial \Omega, \mathbb{R}^{2}\right)$ for variational forms (2.2) and (2.7) to be valid, and $\boldsymbol{g} \in \boldsymbol{H}^{3 / 2}\left(\partial \Omega, \mathbb{R}^{2}\right)$ for error estimates in Theorem 2.3, 2.5 and 2.9 to be true. The discontinuous boundary data $\boldsymbol{g}^{d c}$ certainly poses a problem here. A popular solution to this is to regularize the boundary condition, as what 
is used in the spectral method. However, in finite element approximations, noticing that discrete problems (2.3) and (2.11) are well defined under boundary data $\boldsymbol{g}^{d c}$, people usually use directly $\boldsymbol{g}^{d c} /$ "leaky" or the so-called "non-leaky" boundary condition, in which the velocity on two top corners is set to be $(0,0)^{t}$. To our knowledge, there are no existing error estimates for this type of approximation. The purpose of this paper is to provide an error estimate in this situation using both the velocity-pressure formulation and the pseudostress-velocity formulation under the discontinuous boundary condition.

The main idea of our approach towards the error estimate is to first design a continuous boundary data $\boldsymbol{g}_{\varepsilon}^{c} \in \boldsymbol{H}^{1 / 2}\left(\partial \Omega, \mathbb{R}^{2}\right)$, which is presumably a good approximation to $\boldsymbol{g}^{d c}$. The detailed construction of $\boldsymbol{g}_{\varepsilon}^{c}$ will be given later. Let $\left(\boldsymbol{u}_{\varepsilon}^{c}, p_{\varepsilon}^{c}\right) \in\left(\boldsymbol{H}^{1}\left(\Omega, \mathbb{R}^{2}\right), L^{2}(\Omega) / \mathbb{R}\right)$ be the continuous solution corresponding to this new boundary data $\boldsymbol{g}_{\varepsilon}^{c}$. According to Theorem 2.1.

$$
\left\|\boldsymbol{u}-\boldsymbol{u}_{\varepsilon}^{c}\right\|_{1, r, \Omega}+\left\|p-p_{\varepsilon}^{c}\right\|_{0, r, \Omega} \leq C_{r}\left\|\boldsymbol{g}^{d c}-\boldsymbol{g}_{\varepsilon}^{c}\right\|_{1-1 / r, r, \partial \Omega} \quad \text { for } 1<r<2 .
$$

Then the variational form is discretized using the continuous boundary data $\boldsymbol{g}_{\varepsilon}^{c}$. One important observation is that, for properly designed $\boldsymbol{g}_{\varepsilon}^{c}$, the discrete system will not see the difference between $\boldsymbol{g}^{d c}$ and $\boldsymbol{g}_{\varepsilon}^{c}$. In other words, discrete systems with boundary data $\boldsymbol{g}^{d c}$ and $\boldsymbol{g}_{\varepsilon}^{c}$ are identical. Then the error estimate can be done by using the triangle inequality. Here, one essential step is to choose $\boldsymbol{g}_{\varepsilon}^{c}$ carefully. Our definition of $\boldsymbol{g}_{\varepsilon}^{c}$ is given in the following.

Simple calculation shows that a fourth degree polynomial $f(x)$ on $x \in[0,1]$ can be uniquely determined by the following conditions:

(1) $f(0)=0, f(1)=1$ and $f^{\prime}(0)=f^{\prime}(1)=0$;

(2) for the velocity-pressure formulation, set $f(1 / 2)=1$; for the pseudostressvelocity formulation, set $\int_{0}^{1} f(x) d x=1$.

Notice that $f(x)$ varies for different formulations. However, this will not affect later analysis. The top boundary of $\Omega,[0, a] \times b$, is divided into segments by the mesh $\mathcal{T}_{h}$ (see Figure 1). Consider the two segments which contain the two upper corners $(0, b)$ and $(a, b)$. Without loss of generality, we assume these two segments have the same length $\varepsilon$. It is clear that $\varepsilon=O(h)$. Denote

$$
\Gamma_{\varepsilon}^{1}=\{x \in[0, \varepsilon], y=b\}, \quad \Gamma_{\varepsilon}^{2}=\{x \in[a-\varepsilon, a], y=b\}
$$

as shown in Figure 1 and set $\Gamma_{\varepsilon}=\Gamma_{\varepsilon}^{1} \cup \Gamma_{\varepsilon}^{2}$. Define

$$
\boldsymbol{g}_{\varepsilon}^{c}= \begin{cases}(1,0)^{t} & \text { on } \Gamma_{1} \backslash \Gamma_{\varepsilon}, \\ (f(x / \varepsilon), 0)^{t} & \text { on } \Gamma_{\varepsilon}^{1} \\ (f((a-x) / \varepsilon), 0)^{t} & \text { on } \Gamma_{\varepsilon}^{2} \\ (0,0)^{t} & \text { on } \Gamma_{0}\end{cases}
$$

It is easy to see that $\boldsymbol{g}_{\varepsilon}^{c} \in \boldsymbol{H}^{2}\left(\partial \Omega, \mathbb{R}^{2}\right)$. Furthermore, discrete solutions using the boundary data $\boldsymbol{g}_{\varepsilon}^{c}$ and $\boldsymbol{g}^{d c}$ are exactly the same in the pseudostress-velocity formulation (2.11). Indeed, the boundary term $\left\langle\boldsymbol{g}_{\varepsilon}^{c}, \boldsymbol{\tau}_{h} \boldsymbol{n}\right\rangle_{\partial \Omega}$ is just proportional to the line integral of $\boldsymbol{g}_{\varepsilon}^{c}$ along boundary edges, since the normal components of the lowest order $R T$ element on the boundary are piecewise constants. By the definition of $\boldsymbol{g}_{\varepsilon}^{c}$, it is easy to verify that

$$
\left\langle\boldsymbol{g}_{\varepsilon}^{c}, \boldsymbol{\tau}_{h} \boldsymbol{n}\right\rangle_{\partial \Omega}=\left\langle\boldsymbol{g}^{d c}, \boldsymbol{\tau}_{h} \boldsymbol{n}\right\rangle_{\partial \Omega} \quad \text { for all } \boldsymbol{\tau}_{h} \in \boldsymbol{\Sigma}_{h} .
$$


In the velocity-pressure formulation (2.3), discrete solutions are the same for $\boldsymbol{g}_{\varepsilon}^{c}$ and $\boldsymbol{g}^{d c}$ only when the nodal value interpolation is used to approximate the boundary data. For higher order finite elements, one can design suitable $\boldsymbol{g}_{\varepsilon}^{c}$ similarly.

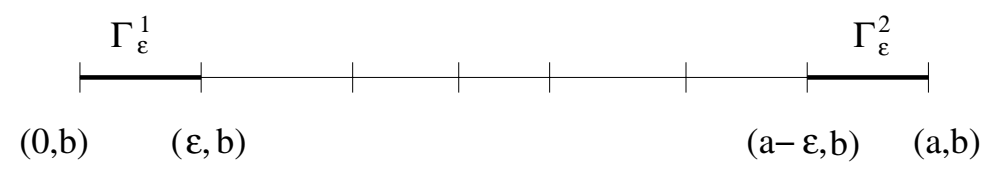

FiguRE 1 . The top boundary of $\Omega$.

Remark 3.1. The continuous boundary data $\boldsymbol{g}_{\varepsilon}^{c}$ is designed purely for theoretical analysis. In numerical simulation, it is expected that only $\boldsymbol{g}^{d c}$ will be used. This is clear in the case of the pseudostress-velocity formulation. However, in the case of the velocity-pressure formulation, this is only true if one uses the nodal value interpolation to approximate the boundary data.

To derive error estimates in fractional Sobolev norms, some technical inequalities will be needed. The proof of the following lemma will be given in Appendix B.

Lemma 3.2. For $1<r<2$ and $0<\varepsilon<1 / 2$,

$$
\begin{array}{ll}
\int_{-1}^{0} \int_{0}^{\varepsilon} \frac{y^{r}}{(y-x)^{r}} d y d x \leq-2 \varepsilon^{2} \ln \varepsilon, & \int_{0}^{\varepsilon} \int_{\varepsilon}^{1} \frac{(\varepsilon-y)^{r}}{(x-y)^{r}} d x d y \leq-2 \varepsilon^{2} \ln \varepsilon \\
\int_{-1}^{0} \int_{0}^{\varepsilon} \frac{1}{(y-x)^{r}} d y d x \leq \frac{-4 \varepsilon^{2-r} \ln \varepsilon}{2-r}, & \int_{0}^{\varepsilon} \int_{\varepsilon}^{1} \frac{1}{(x-y)^{r}} d x d y \leq \frac{-4 \varepsilon^{2-r} \ln \varepsilon}{2-r} .
\end{array}
$$

Furthermore, for $r=2$ and $0<\varepsilon<1 / 2$,

$$
\int_{-1}^{0} \int_{0}^{\varepsilon} \frac{y^{2}}{(y-x)^{2}} d y d x \leq \varepsilon^{2} / 2, \quad \int_{0}^{\varepsilon} \int_{\varepsilon}^{1} \frac{(\varepsilon-y)^{2}}{(x-y)^{2}} d x d y \leq \varepsilon^{2} / 2 .
$$

Now we are able to estimate the fractional Sobolev norm of $\boldsymbol{g}^{d c}-\boldsymbol{g}_{\varepsilon}^{c}$ and $\boldsymbol{g}_{\varepsilon}^{c}$ :

Lemma 3.3. For $0<\varepsilon<1 / 2$ and $1<r<2$,

$$
\left\|\boldsymbol{g}^{d c}-\boldsymbol{g}_{\varepsilon}^{c}\right\|_{1-1 / r, r, \partial \Omega} \lesssim\left(\frac{-\varepsilon^{2-r} \ln \varepsilon}{2-r}\right)^{1 / r}
$$

and

$$
\left\|\boldsymbol{g}_{\varepsilon}^{c}\right\|_{2-1 / r, r, \partial \Omega} \lesssim\left(-\varepsilon^{2-2 r} \ln \varepsilon\right)^{1 / r}
$$

For $r=2$,

$$
\left\|\boldsymbol{g}_{\varepsilon}^{c}\right\|_{1 / 2, \partial \Omega} \lesssim(-\ln \varepsilon)^{1 / 2}, \quad\left\|\boldsymbol{g}_{\varepsilon}^{c}\right\|_{3 / 2, \partial \Omega} \lesssim \varepsilon^{-1} \quad \text { and } \quad\left\|\boldsymbol{g}_{\varepsilon}^{c}\right\|_{2, \partial \Omega} \lesssim \varepsilon^{-3 / 2} .
$$

Proof. Let us start from a simplified case. Consider the following discontinuous function defined on $\mathcal{I}=(-1,1)$ :

$$
f_{\varepsilon}(x)= \begin{cases}0 & -1<x<0 \\ 1-f(x / \varepsilon) & 0 \leq x \leq \varepsilon \\ 0 & \varepsilon<x<1\end{cases}
$$


Define $F=\max \left\{1+\max _{0 \leq x \leq 1}|f(x)|, \max _{0 \leq x \leq 1}\left|f^{\prime}(x)\right|\right\}$. By the definition of the fractional Sobolev norm, Lemma 3.2 and using the change of variables,

$$
\begin{aligned}
\left\|f_{\varepsilon}\right\|_{1-1 / r, r, \mathcal{I}}^{r}=\int_{0}^{\varepsilon}\left|f_{\varepsilon}(x)\right|^{r} d x+2 \int_{-1}^{0} \int_{0}^{\varepsilon} \frac{|1-f(y / \varepsilon)|^{r}}{|y-x|^{r}} d y d x \\
\quad+2 \int_{0}^{\varepsilon} \int_{\varepsilon}^{1} \frac{|1-f(y / \varepsilon)|^{r}}{|x-y|^{r}} d x d y+\int_{0}^{\varepsilon} \int_{0}^{\varepsilon} \frac{|f(x / \varepsilon)-f(y / \varepsilon)|^{r}}{|x-y|^{r}} d x d y \\
\leq F^{r} \varepsilon+4 F^{r} \frac{-4 \varepsilon^{2-r} \ln \varepsilon}{2-r}+\int_{0}^{1} \int_{0}^{1} \frac{|f(x)-f(y)|^{r}}{|x-y|^{r}} \varepsilon^{2-r} d x d y \\
\leq F^{r}\left(\varepsilon-16 \frac{\varepsilon^{2-r} \ln \varepsilon}{2-r}+\varepsilon^{2-r}\right) \lesssim \frac{-\varepsilon^{2-r} \ln \varepsilon}{2-r} .
\end{aligned}
$$

The last step comes from the facts that $1<r<2$ and that $\varepsilon$ is small. Notice that

$$
\boldsymbol{g}^{d c}-\boldsymbol{g}_{\varepsilon}^{c}= \begin{cases}(1-f(x / \varepsilon), 0)^{t} & \text { on } \Gamma_{\varepsilon}^{1}, \\ (1-f((a-x) / \varepsilon), 0)^{t} & \text { on } \Gamma_{\varepsilon}^{2}, \\ \mathbf{0} & \text { elsewhere. }\end{cases}
$$

Hence inequality (3.4) can be proved by the same process as above.

To prove inequality (3.5), we define a continuous function

$$
k_{\varepsilon}(x)= \begin{cases}0 & -1<x<0 \\ f(x / \varepsilon) & 0 \leq x \leq \varepsilon \\ 1 & \varepsilon<x<1\end{cases}
$$

Let $G=\max _{0<x<1}\left|f^{\prime \prime}(x)\right|$. Notice that $k_{\varepsilon}^{\prime}(x)$ is a continuous piecewise polynomial and vanishes for $-1<x \leq 0$ and $\varepsilon \leq x<1$. Hence it is Lipschitz continuous with

$$
\left|k_{\varepsilon}^{\prime}(y)-k_{\varepsilon}^{\prime}(x)\right| \leq \max _{0<t<\varepsilon}\left(\left|k_{\varepsilon}^{\prime \prime}(t)\right|\right)|y-x| \leq \frac{G}{\varepsilon^{2}}|y-x| \quad \text { for }-1<x, y<1 .
$$

Consequently, for $0<y<\varepsilon$,

$$
\begin{aligned}
& \left|k_{\varepsilon}^{\prime}(y)\right|=\left|k_{\varepsilon}^{\prime}(y)-k_{\varepsilon}^{\prime}(0)\right| \leq \frac{G}{\varepsilon^{2}}|y|, \\
& \left|k_{\varepsilon}^{\prime}(y)\right|=\left|k_{\varepsilon}^{\prime}(y)-k_{\varepsilon}^{\prime}(\varepsilon)\right| \leq \frac{G}{\varepsilon^{2}}|\varepsilon-y| .
\end{aligned}
$$

Then by Lemma 3.2 ,

$$
\begin{aligned}
& \left\|k_{\varepsilon}\right\|_{2-1 / r, r, \mathcal{I}}^{r}=\left\|k_{\varepsilon}\right\|_{1, r, \mathcal{I}}^{r}+2 \int_{-1}^{0} \int_{0}^{\varepsilon} \frac{\left|k_{\varepsilon}^{\prime}(y)\right|^{r}}{|x-y|^{r}} d y d x \\
& \quad+2 \int_{0}^{\varepsilon} \int_{\varepsilon}^{1} \frac{\left|k_{\varepsilon}^{\prime}(y)\right|^{r}}{|x-y|^{r}} d x d y+\int_{0}^{\varepsilon} \int_{0}^{\varepsilon} \frac{\left|k_{\varepsilon}^{\prime}(y)-k_{\varepsilon}^{\prime}(x)\right|^{r}}{|x-y|^{r}} d x d y \\
& \quad \leq\left(1+F^{r} \varepsilon+F^{r} \varepsilon^{1-r}\right)+4 \frac{G^{r}}{\varepsilon^{2 r}}\left(-2 \varepsilon^{2} \ln \varepsilon\right)+G^{r} \varepsilon^{2-2 r} \\
& \quad \lesssim-\varepsilon^{2-2 r} \ln \varepsilon .
\end{aligned}
$$

This completes the proof for inequality (3.5).

Finally, the error estimate for $\left\|\boldsymbol{g}_{\varepsilon}^{c}\right\|_{1 / 2, \partial \Omega},\left\|\boldsymbol{g}_{\varepsilon}^{c}\right\|_{3 / 2, \partial \Omega}$ and $\left\|\boldsymbol{g}_{\varepsilon}^{c}\right\|_{2, \partial \Omega}$ can be obtained similarly by using Lemma 3.2

The following two theorems give the detailed error estimates for different finite element approximations to the driven cavity problem. 
Theorem 3.4. Let $(\boldsymbol{u}, p)$ be the solution to problem (2.1) and let $\left(\boldsymbol{u}_{h}, p_{h}\right)$ be the solution to problem (2.3) with $\boldsymbol{f}=\mathbf{0}$ and $\boldsymbol{g}=\boldsymbol{g}^{d c}$. Assume that nodal value approximation is used in treating the boundary data for $\boldsymbol{u}_{h}$. Then, for $1<r<2$, we have

$$
\begin{gathered}
\left\|\boldsymbol{u}-\boldsymbol{u}_{h}\right\|_{2-2 / r, \Omega} \lesssim C_{r}\left(\frac{h^{2-3 r / 2}}{2-r}\right)^{1 / r}, \\
\left\|\boldsymbol{u}-\boldsymbol{u}_{h}\right\|_{0, \Omega} \lesssim \inf _{1<r<2} C_{r}\left(\frac{h^{2-3 r / 2}}{2-r}\right)^{1 / r} .
\end{gathered}
$$

If $\left(\boldsymbol{u}_{h}, p_{h}\right)$ is defined with the boundary data to be the $\boldsymbol{H}^{3 / 2}$ projection of $\boldsymbol{g}_{\varepsilon}^{c}$, then it gives the optimal error estimate

$$
\begin{gathered}
\left\|\boldsymbol{u}-\boldsymbol{u}_{h}\right\|_{2-2 / r, \Omega} \lesssim C_{r}\left(\frac{-h^{2-r} \ln h}{2-r}\right)^{1 / r}, \\
\left\|\boldsymbol{u}-\boldsymbol{u}_{h}\right\|_{0, \Omega} \lesssim \inf _{1<r<2} C_{r}\left(\frac{-h^{2-r} \ln h}{2-r}\right)^{1 / r} .
\end{gathered}
$$

Proof. Let $\left(\boldsymbol{u}_{\varepsilon}^{c}, p_{\varepsilon}^{c}\right)$ be the solution to problem (2.1) with $\boldsymbol{f}=\mathbf{0}$ and the boundary data $\boldsymbol{g}=\boldsymbol{g}_{\varepsilon}^{c}$. According to Theorem 2.1, $\boldsymbol{u}_{\varepsilon}^{c}$ is in $\boldsymbol{H}^{2}\left(\Omega, \mathbb{R}^{2}\right)$. Notice that $\left(\boldsymbol{u}_{h}, p_{h}\right)$ can also be considered as the solution to problem (2.3) with boundary data set as an interpolant of $\boldsymbol{g}_{\varepsilon}^{c}$. Let $\mathbf{I}_{h}$ be the nodal value interpolation operator from $\boldsymbol{H}^{2}\left(\Omega, \mathbb{R}^{2}\right)$ into $\mathbf{U}_{h}$. Then by the triangle inequality and the Sobolev embedding theorem,

$$
\begin{aligned}
& \left\|\boldsymbol{u}-\boldsymbol{u}_{h}\right\|_{2-2 / r, \Omega} \leq\left\|\boldsymbol{u}-\boldsymbol{u}_{\varepsilon}^{c}\right\|_{2-2 / r, \Omega}+\left\|\boldsymbol{u}_{\varepsilon}^{c}-\mathbf{I}_{h} \boldsymbol{u}_{\varepsilon}^{c}\right\|_{2-2 / r, \Omega}+\left\|\mathbf{I}_{h} \boldsymbol{u}_{\varepsilon}^{c}-\boldsymbol{u}_{h}\right\|_{2-2 / r, \Omega} \\
& \quad \lesssim\left\|\boldsymbol{u}-\boldsymbol{u}_{\varepsilon}^{c}\right\|_{1, r, \Omega}+\left\|\boldsymbol{u}_{\varepsilon}^{c}-\mathbf{I}_{h} \boldsymbol{u}_{\varepsilon}^{c}\right\|_{1, r, \Omega}+\left\|\mathbf{I}_{h} \boldsymbol{u}_{\varepsilon}^{c}-\boldsymbol{u}_{h}\right\|_{2-2 / r, \Omega}
\end{aligned}
$$

Next, it follows from the approximation property of $\mathbf{I}_{h}, 3$, Theorem 2.1, 2.3, 2.5. the inverse inequality, and the triangle inequality that, for $1<r<2$, we have

$$
\left\|\boldsymbol{u}_{\varepsilon}^{c}-\mathbf{I}_{h} \boldsymbol{u}_{\varepsilon}^{c}\right\|_{1, r, \Omega} \lesssim h\left\|\boldsymbol{u}_{\varepsilon}^{c}\right\|_{2, r, \Omega} \lesssim h C_{r}\left\|\boldsymbol{g}_{\varepsilon}^{c}\right\|_{2-1 / r, r, \partial \Omega}
$$

and

$$
\begin{aligned}
\left\|\mathbf{I}_{h} \boldsymbol{u}_{\varepsilon}^{c}-\boldsymbol{u}_{h}\right\|_{2-2 / r, \Omega} & \lesssim h^{-2+2 / r}\left\|\mathbf{I}_{h} \boldsymbol{u}_{\varepsilon}^{c}-\boldsymbol{u}_{h}\right\|_{0, \Omega} \\
& \lesssim h^{-2+2 / r}\left(\left\|\mathbf{I}_{h} \boldsymbol{u}_{\varepsilon}^{c}-\boldsymbol{u}_{\varepsilon}^{c}\right\|_{0, \Omega}+\left\|\boldsymbol{u}_{\varepsilon}^{c}-\boldsymbol{u}_{h}\right\|_{0, \Omega}\right) \\
& \lesssim h^{2 / r}\left(\left\|\boldsymbol{u}_{\varepsilon}^{c}\right\|_{2, \Omega}+\left\|p_{\varepsilon}^{c}\right\|_{1, \Omega}+\left\|\boldsymbol{g}_{\varepsilon}^{c}\right\|_{2, \partial \Omega}\right) \\
& \lesssim h^{2 / r}\left\|\boldsymbol{g}_{\varepsilon}^{c}\right\|_{2, \partial \Omega} .
\end{aligned}
$$

Combining the above, Theorem 2.1 and Lemma 3.3 gives

$$
\begin{aligned}
\| \boldsymbol{u}- & \boldsymbol{u}_{h}\left\|_{2-2 / r, \Omega} \lesssim\right\| \boldsymbol{u}-\boldsymbol{u}_{\varepsilon}^{c}\left\|_{1, r, \Omega}+\right\| \boldsymbol{u}_{\varepsilon}^{c}-\mathbf{I}_{h} \boldsymbol{u}_{\varepsilon}^{c}\left\|_{1, r, \Omega}+\right\| \mathbf{I}_{h} \boldsymbol{u}_{\varepsilon}^{c}-\boldsymbol{u}_{h} \|_{2-2 / r, \Omega} \\
& \lesssim C_{r}\left\|\boldsymbol{g}^{d c}-\boldsymbol{g}_{\varepsilon}^{c}\right\|_{1-1 / r, r, \partial \Omega}+h C_{r}\left\|\boldsymbol{g}_{\varepsilon}^{c}\right\|_{2-1 / r, r, \partial \Omega}+h^{2 / r}\left\|\boldsymbol{g}_{\varepsilon}^{c}\right\|_{2, \partial \Omega} \\
& \lesssim C_{r}\left(\frac{-\varepsilon^{2-r} \ln \varepsilon}{2-r}\right)^{1 / r}+h C_{r}\left(-\varepsilon^{2-2 r} \ln \varepsilon\right)^{1 / r}+h^{2 / r} \varepsilon^{-3 / 2} \\
& \lesssim C_{r}\left(\frac{h^{2-3 r / 2}}{2-r}\right)^{1 / r} .
\end{aligned}
$$

The last step follows from the fact that $\varepsilon=O(h)$. This completes the proof for inequality (3.6). Inequality (3.7) is an immediate consequence of inequality (3.6). 
In the case of using the $\boldsymbol{H}^{3 / 2}$ projection to approximate the boundary data $\boldsymbol{g}_{\varepsilon}^{c}$, all other estimates still hold except that $\left\|\mathbf{I}_{h} \boldsymbol{u}_{\varepsilon}^{c}-\boldsymbol{u}_{h}\right\|_{2-2 / r, \Omega}$ becomes

$$
\begin{aligned}
\left\|\mathbf{I}_{h} \boldsymbol{u}_{\varepsilon}^{c}-\boldsymbol{u}_{h}\right\|_{2-2 / r, \Omega} & \lesssim h^{-2+2 / r}\left(\left\|\mathbf{I}_{h} \boldsymbol{u}_{\varepsilon}^{c}-\boldsymbol{u}_{\varepsilon}^{c}\right\|_{0, \Omega}+\left\|\boldsymbol{u}_{\varepsilon}^{c}-\boldsymbol{u}_{h}\right\|_{0, \Omega}\right) \\
& \lesssim h^{2 / r}\left(\left\|\boldsymbol{u}_{\varepsilon}^{c}\right\|_{2, \Omega}+\left\|p_{\varepsilon}^{c}\right\|_{1, \Omega}+\left\|\boldsymbol{g}_{\varepsilon}^{c}\right\|_{3 / 2, \partial \Omega}\right) \\
& \lesssim h^{2 / r}\left\|\boldsymbol{g}_{\varepsilon}^{c}\right\|_{3 / 2, \partial \Omega} \\
& \lesssim h^{2 / r} \varepsilon^{-1} .
\end{aligned}
$$

Hence

$$
\begin{aligned}
\left\|\boldsymbol{u}-\boldsymbol{u}_{h}\right\|_{2-2 / r, \Omega} & \lesssim C_{r}\left(\frac{-\varepsilon^{2-r} \ln \varepsilon}{2-r}\right)^{1 / r}+h C_{r}\left(-\varepsilon^{2-2 r} \ln \varepsilon\right)^{1 / r}+h^{2 / r} \varepsilon^{-1} \\
& \lesssim C_{r}\left(\frac{-h^{2-r} \ln h}{2-r}\right)^{1 / r} .
\end{aligned}
$$

This completes the proof for inequality (3.8). Inequality (3.9) follows immediately.

Theorem 3.5. Let $(\boldsymbol{\sigma}, \boldsymbol{u})$ be the solution to problem (2.6) and let $\left(\boldsymbol{\sigma}_{h}, \boldsymbol{u}_{h}\right)$ be the solution to problem (2.11) with $\boldsymbol{f}=\mathbf{0}$ and $\boldsymbol{g}=\boldsymbol{g}^{d c}$. Then

$$
\left\|\boldsymbol{u}-\boldsymbol{u}_{h}\right\|_{0, \Omega} \lesssim \inf _{1<r<2} C_{r}\left(\frac{-h^{2-r} \ln h}{2-r}\right)^{1 / r}
$$

Proof. Let $\left(\boldsymbol{\sigma}_{\varepsilon}^{c}, \boldsymbol{u}_{\varepsilon}^{c}\right)$ be the solution to problem (2.6) with $\boldsymbol{f}=\mathbf{0}$ and the boundary data $\boldsymbol{g}=\boldsymbol{g}_{\varepsilon}^{c}$. Then by the triangle inequality, Theorem 2.1, Theorem 2.9, and Lemma 3.3

$$
\begin{aligned}
\left\|\boldsymbol{u}-\boldsymbol{u}_{h}\right\|_{0, \Omega} & \leq\left\|\boldsymbol{u}-\boldsymbol{u}_{\varepsilon}^{c}\right\|_{0, \Omega}+\left\|\boldsymbol{u}_{\varepsilon}^{c}-\boldsymbol{u}_{h}\right\|_{0, \Omega} \\
& \lesssim C_{r}\left\|\boldsymbol{g}^{d c}-\boldsymbol{g}_{\varepsilon}^{c}\right\|_{1-1 / r, r, \partial \Omega}+h\left\|\boldsymbol{u}_{\varepsilon}^{c}\right\|_{1, \Omega}+h^{2}\left\|\boldsymbol{\sigma}_{\varepsilon}^{c}\right\|_{1, \Omega} \\
& \lesssim C_{r}\left(\frac{-\varepsilon^{2-r} \ln \varepsilon}{2-r}\right)^{1 / r}+h\left\|\boldsymbol{g}_{\varepsilon}^{c}\right\|_{1 / 2, \partial \Omega}+h^{2}\left\|\boldsymbol{g}_{\varepsilon}^{c}\right\|_{3 / 2, \partial \Omega} \\
& \lesssim C_{r}\left(\frac{-\varepsilon^{2-r} \ln \varepsilon}{2-r}\right)^{1 / r}+h(-\ln \varepsilon)^{1 / 2}+h^{2} \varepsilon^{-1} \\
& \lesssim C_{r}\left(\frac{-h^{2-r} \ln h}{2-r}\right)^{1 / r} .
\end{aligned}
$$

The last step follows from the fact that $\varepsilon=O(h)$.

Finally, we compare the results of Theorem 3.4 and Theorem 3.5. Inequalities (3.7), (3.9) and (3.10) describe the approximating property for, respectively, the velocity-pressure formulation with nodal-value interpolation on the boundary data, the velocity-pressure formulation with $\boldsymbol{H}^{3 / 2}$ projection on the boundary data, and the pseudostress-velocity formulation. Notice all three error bounds have the form

$$
\left\|\boldsymbol{u}-\boldsymbol{u}_{h}\right\|_{0, \Omega} \lesssim \inf _{1<r<2} C_{r} \phi(r)
$$


where $\phi(r)=\left(\frac{h^{2-3 r / 2}}{2-r}\right)^{1 / r}$ for inequality (3.7) and $\phi(r)=\left(\frac{-h^{2-r} \ln h}{2-r}\right)^{1 / r}$ for other cases. It can be shown that $\phi(r)$ takes its infimum at $r=1$ and

$$
\phi(1)= \begin{cases}h^{1 / 2} & \text { for inequality (3.7) } \\ -h \ln h & \text { for other situations. }\end{cases}
$$

However, since $C_{r}$ may not be bounded as $r$ goes to 1 , we want to set $r=1+\delta$ instead of 1 , where $\delta$ is a small positive number. Because $C_{r}$ does not depend on $h$, one can still get an error estimate for $\left\|\boldsymbol{u}-\boldsymbol{u}_{h}\right\|_{0, \Omega}$ of order close to $O\left(h^{1 / 2}\right)$ for inequality (3.7) or $O(-h \ln h)$ for other situations, although it may contain a large coefficient $C_{1+\delta}$. The loss of half an order of $h$ for inequality (3.7) comes from using the nodal-value interpolation on the boundary data.

Recall that the exact velocity $\boldsymbol{u}$ for the two-dimensional Stokes driven cavity problem is in $W^{1, r}\left(\Omega, \mathbb{R}^{2}\right)$ for all $1<r<2$. By the Sobolev embedding theorem [17, the exact velocity $\boldsymbol{u}$ is in $\boldsymbol{H}^{1-\varepsilon}\left(\Omega, \mathbb{R}^{2}\right)$ for $\varepsilon$ arbitrarily small. Therefore, we expect the optimal bound of $\left\|\boldsymbol{u}-\boldsymbol{u}_{h}\right\|_{0, \Omega}$ will be close to $O(h)$, but not better than that, even when higher order finite element spaces are used. Judging from this, an error of order close to $C_{1+\delta} O(-h \ln h)$, for inequalities (3.9) and (3.10), seems reasonable. However, we are not sure whether improvements can be done on the $C_{1+\delta}$ part or not.

\section{Appendix A. Proof of Theorem 2.9}

Let $(\boldsymbol{\sigma}, \boldsymbol{u})$ be the solution to problem (2.7) and let $\left(\boldsymbol{\sigma}_{h}, \boldsymbol{u}_{h}\right)$ be the solution to problem (2.11). It is easy to see from Lemma 2.8 that

$$
\operatorname{div} \Pi_{h} \sigma=\mathbf{P}_{h} \operatorname{div} \sigma=\operatorname{div} \sigma_{h} .
$$

By subtracting equations (2.7) from (2.11) and using Lemma 2.8, one can derive that

$$
\begin{aligned}
\left\|\mathcal{A}^{1 / 2}\left(\boldsymbol{\sigma}-\boldsymbol{\sigma}_{h}\right)\right\|_{0, \Omega}^{2}=\left(\mathcal{A}^{1 / 2}\left(\boldsymbol{\sigma}-\boldsymbol{\sigma}_{h}\right), \mathcal{A}^{1 / 2}\left(\boldsymbol{\sigma}-\Pi_{h} \boldsymbol{\sigma}\right)\right)+\left(\mathcal{A}\left(\boldsymbol{\sigma}-\boldsymbol{\sigma}_{h}\right), \Pi_{h} \boldsymbol{\sigma}-\boldsymbol{\sigma}_{h}\right) \\
\quad \lesssim h\left\|\mathcal{A}^{1 / 2}\left(\boldsymbol{\sigma}-\boldsymbol{\sigma}_{h}\right)\right\|_{0, \Omega}|\boldsymbol{\sigma}|_{1, \Omega}-\left(\operatorname{div}\left(\Pi_{h} \boldsymbol{\sigma}-\boldsymbol{\sigma}_{h}\right), \boldsymbol{u}-\boldsymbol{u}_{h}\right) \\
\quad=h\left\|\mathcal{A}^{1 / 2}\left(\boldsymbol{\sigma}-\boldsymbol{\sigma}_{h}\right)\right\|_{0, \Omega}|\boldsymbol{\sigma}|_{1, \Omega} .
\end{aligned}
$$

Therefore

$$
\left\|\mathcal{A}^{1 / 2}\left(\boldsymbol{\sigma}-\boldsymbol{\sigma}_{h}\right)\right\|_{0, \Omega} \lesssim h|\boldsymbol{\sigma}|_{1, \Omega}
$$

Notice that

$$
\begin{aligned}
\left\|\operatorname{div}\left(\boldsymbol{\sigma}-\boldsymbol{\sigma}_{h}\right)\right\|_{-1, \Omega}=\sup _{\boldsymbol{v} \in \boldsymbol{H}_{0}^{1}\left(\Omega, \mathbb{R}^{2}\right)} \frac{\left(\operatorname{div}\left(\boldsymbol{\sigma}-\boldsymbol{\sigma}_{h}\right), \boldsymbol{v}\right)}{\|\boldsymbol{v}\|_{1, \Omega}} \\
\quad=\sup _{\boldsymbol{v} \in \boldsymbol{H}_{0}^{1}\left(\Omega, \mathbb{R}^{2}\right)} \frac{\left(\operatorname{div}\left(\boldsymbol{\sigma}-\boldsymbol{\sigma}_{h}\right), \boldsymbol{v}-\mathbf{P}_{h} \boldsymbol{v}\right)}{\|\boldsymbol{v}\|_{1, \Omega}} \lesssim h\left\|\operatorname{div}\left(\boldsymbol{\sigma}-\boldsymbol{\sigma}_{h}\right)\right\|_{0, \Omega} .
\end{aligned}
$$

Hence by Lemma 2.7

$$
\begin{gathered}
\left\|\boldsymbol{\sigma}-\boldsymbol{\sigma}_{h}\right\|_{0, \Omega} \lesssim\left\|\mathcal{A}^{1 / 2}\left(\boldsymbol{\sigma}-\boldsymbol{\sigma}_{h}\right)\right\|_{0, \Omega}+\left\|\operatorname{div}\left(\boldsymbol{\sigma}-\boldsymbol{\sigma}_{h}\right)\right\|_{-1, \Omega} \\
\lesssim h\left(|\boldsymbol{\sigma}|_{1, \Omega}+\left\|\operatorname{div}\left(\boldsymbol{\sigma}-\boldsymbol{\sigma}_{h}\right)\right\|_{0, \Omega}\right) \lesssim h|\boldsymbol{\sigma}|_{1, \Omega} .
\end{gathered}
$$

Let $\boldsymbol{\tau}$ satisfy $\operatorname{div} \boldsymbol{\tau}=\mathbf{P}_{h} \boldsymbol{u}-\boldsymbol{u}_{h}$ and $\|\boldsymbol{\tau}\|_{1, \Omega} \lesssim\left\|\mathbf{P}_{h} \boldsymbol{u}-\boldsymbol{u}_{h}\right\|_{0, \Omega} \lesssim\left\|\boldsymbol{u}-\boldsymbol{u}_{h}\right\|_{0, \Omega}$. Such a $\boldsymbol{\tau}$ can be easily constructed by solving a Stokes problem using the force function 
$\mathbf{P}_{h} \boldsymbol{u}-\boldsymbol{u}_{h}$. Clearly, $\operatorname{div} \Pi_{h} \boldsymbol{\tau}=\operatorname{div} \boldsymbol{\tau}$. Again by subtracting equations (2.7) from (2.11) and using Lemma 2.8, we get

$$
\begin{aligned}
\left\|\boldsymbol{u}-\boldsymbol{u}_{h}\right\|_{0, \Omega}^{2} & =\left(\boldsymbol{u}-\boldsymbol{u}_{h}, \boldsymbol{u}-\mathbf{P}_{h} \boldsymbol{u}\right)+\left(\boldsymbol{u}-\boldsymbol{u}_{h}, \mathbf{P}_{h} \boldsymbol{u}-\boldsymbol{u}_{h}\right) \\
& \lesssim h\left\|\boldsymbol{u}-\boldsymbol{u}_{h}\right\|_{0, \Omega}|\boldsymbol{u}|_{1, \Omega}+\left(\boldsymbol{u}-\boldsymbol{u}_{h}, \operatorname{div} \Pi_{h} \boldsymbol{\tau}\right) \\
& =h\left\|\boldsymbol{u}-\boldsymbol{u}_{h}\right\|_{0, \Omega}|\boldsymbol{u}|_{1, \Omega}-\left(\mathcal{A}\left(\boldsymbol{\sigma}-\boldsymbol{\sigma}_{h}\right), \Pi_{h} \boldsymbol{\tau}\right) \\
& \lesssim h\left\|\boldsymbol{u}-\boldsymbol{u}_{h}\right\|_{0, \Omega}|\boldsymbol{u}|_{1, \Omega}+h|\boldsymbol{\sigma}|_{1, \Omega}\|\boldsymbol{\tau}\|_{1, \Omega} \\
& \lesssim h\left\|\boldsymbol{u}-\boldsymbol{u}_{h}\right\|_{0, \Omega}\left(|\boldsymbol{u}|_{1, \Omega}+|\boldsymbol{\sigma}|_{1, \Omega}\right) .
\end{aligned}
$$

This completes the proof of inequality (2.12).

Next we prove inequality (2.13). For $\boldsymbol{w} \in \mathbf{V}$, define $\left(\boldsymbol{\sigma}_{\boldsymbol{w}}, \boldsymbol{u}_{\boldsymbol{w}}\right)$ by

$$
\begin{cases}\left(\mathcal{A} \boldsymbol{\sigma}_{\boldsymbol{w}}, \boldsymbol{\tau}\right)+\left(\operatorname{div} \boldsymbol{\tau}, \boldsymbol{u}_{\boldsymbol{w}}\right)=0 & \text { for all } \boldsymbol{\tau} \in \boldsymbol{\Sigma} \\ \left(\operatorname{div} \boldsymbol{\sigma}_{\boldsymbol{w}}, \boldsymbol{v}\right)=(\boldsymbol{w}, \boldsymbol{v}) & \text { for all } \boldsymbol{v} \in \mathbf{V}\end{cases}
$$

Then

$$
\begin{aligned}
\left(\boldsymbol{w}, \boldsymbol{u}_{h}\right. & \left.-\mathbf{P}_{h} \boldsymbol{u}\right)=\left(\operatorname{div} \boldsymbol{\sigma}_{\boldsymbol{w}}, \boldsymbol{u}_{h}-\mathbf{P}_{h} \boldsymbol{u}\right) \\
& =\left(\operatorname{div} \Pi_{h} \boldsymbol{\sigma}_{\boldsymbol{w}}, \boldsymbol{u}_{h}-\boldsymbol{u}\right)=\left(A\left(\boldsymbol{\sigma}-\boldsymbol{\sigma}_{h}\right), \Pi_{h} \boldsymbol{\sigma}_{\boldsymbol{w}}\right) \\
& =\left(A\left(\boldsymbol{\sigma}-\boldsymbol{\sigma}_{h}\right), \Pi_{h} \boldsymbol{\sigma}_{\boldsymbol{w}}-\boldsymbol{\sigma}_{\boldsymbol{w}}\right)+\left(A\left(\boldsymbol{\sigma}-\boldsymbol{\sigma}_{h}\right), \boldsymbol{\sigma}_{\boldsymbol{w}}\right) \\
& =\left(A\left(\boldsymbol{\sigma}-\boldsymbol{\sigma}_{h}\right), \Pi_{h} \boldsymbol{\sigma}_{\boldsymbol{w}}-\boldsymbol{\sigma}_{\boldsymbol{w}}\right)-\left(\operatorname{div}\left(\boldsymbol{\sigma}-\boldsymbol{\sigma}_{h}\right), \boldsymbol{u}_{\boldsymbol{w}}\right) \\
& =\left(A\left(\boldsymbol{\sigma}-\boldsymbol{\sigma}_{h}\right), \Pi_{h} \boldsymbol{\sigma}_{\boldsymbol{w}}-\boldsymbol{\sigma}_{\boldsymbol{w}}\right) .
\end{aligned}
$$

The last step comes from the assumption that $\operatorname{div} \boldsymbol{\sigma}=\operatorname{div} \boldsymbol{\sigma}_{h}$. Hence

$$
\begin{aligned}
\| \boldsymbol{u}_{h} & -\mathbf{P}_{h} \boldsymbol{u} \|_{0, \Omega}=\sup _{\boldsymbol{w} \in \mathbf{V}} \frac{\left(\boldsymbol{w}, \boldsymbol{u}_{h}-\mathbf{P}_{h} \boldsymbol{u}\right)}{\|\boldsymbol{w}\|_{0, \Omega}} \\
& =\sup _{\boldsymbol{w} \in \mathbf{V}} \frac{\left(A\left(\boldsymbol{\sigma}-\boldsymbol{\sigma}_{h}\right), \Pi_{h} \boldsymbol{\sigma}_{\boldsymbol{w}}-\boldsymbol{\sigma}_{\boldsymbol{w}}\right)}{\|\boldsymbol{w}\|_{0, \Omega}} \lesssim \sup _{\boldsymbol{w} \in \mathbf{V}} \frac{h^{2}|\boldsymbol{\sigma}|_{1, \Omega}\left|\boldsymbol{\sigma}_{\boldsymbol{w}}\right|_{1, \Omega}}{\|\boldsymbol{w}\|_{0, \Omega}} \\
& \lesssim h^{2}|\boldsymbol{\sigma}|_{1, \Omega} .
\end{aligned}
$$

Finally, by using the triangular inequality and the property of $\mathbf{L}^{2}$ projection,

$$
\left\|\boldsymbol{u}-\boldsymbol{u}_{h}\right\|_{0, \Omega} \leq\left\|\boldsymbol{u}-\mathbf{P}_{h} \boldsymbol{u}\right\|_{0, \Omega}+\left\|\boldsymbol{u}_{h}-\mathbf{P}_{h} \boldsymbol{u}\right\|_{0, \Omega} \lesssim h|\boldsymbol{u}|_{1, \Omega}+h^{2}|\boldsymbol{\sigma}|_{1, \Omega} .
$$

This completes the proof of inequality (2.13).

\section{Appendix B. Proof of Lemma 3.2}

Lemma B.1. Let $0<\varepsilon<1 / 2$ and $1<r<2$, then

$$
\varepsilon^{2-r}-(\varepsilon+1)^{2-r}+1 \leq\left(\frac{3}{2 \ln 2}+1\right)(r-1) \varepsilon^{2-r}(-\ln \varepsilon) .
$$

Proof. Define function

$$
f(\varepsilon, r)=\varepsilon^{2-r}-(\varepsilon+1)^{2-r}+1 .
$$


Clearly, $f(\varepsilon, r)$ is differentiable in $(0,1 / 2) \times[1,2)$. Notice that $f(\varepsilon, 1)=0$, then

$$
\begin{aligned}
f(\varepsilon, r) & =f(\varepsilon, r)-f(\varepsilon, 1) \\
& \leq \sup _{1 \leq \xi \leq r}\left|\frac{\partial f}{\partial r}(\varepsilon, \xi)\right|(r-1) \\
& =(r-1) \sup _{1 \leq \xi \leq r}\left[-\varepsilon^{2-\xi} \ln \varepsilon+(\varepsilon+1)^{2-\xi} \ln (\varepsilon+1)\right] \\
& \leq(r-1)\left[-\varepsilon^{2-r} \ln \varepsilon+(\varepsilon+1) \ln (\varepsilon+1)\right] \\
& \leq\left(\frac{3}{2 \ln 2}+1\right)(r-1) \varepsilon^{2-r}(-\ln \varepsilon) .
\end{aligned}
$$

Here the last step comes from the fundamental inequality $\ln (1+\varepsilon)<\varepsilon \leq \varepsilon^{2-r}$ and assumptions that $0<\varepsilon<1 / 2$ and $1<r<2$.

Lemma B.2. Assume $0<y<1 / 2$ and $1<r<2$. Then

$$
y^{1-r}-(y+1)^{1-r} \leq 2(r-1) y^{1-r}(-\ln y) .
$$

Proof. Define $f(y, r)=y^{1-r}-(y+1)^{1-r}$. It is smooth on $0<y<1 / 2$ and $1 \leq r<2$. Notice that $f(y, 1)=0$. Hence

$$
f(y, r)=f(y, r)-f(y, 1) \leq \sup _{1 \leq \xi \leq r}\left|\frac{\partial f}{\partial r}(y, \xi)\right|(r-1) .
$$

Since for $0<y<1 / 2$ and $1 \leq r<2$,

$$
\frac{\partial^{2} f}{\partial r^{2}}(y, r)=\frac{\ln ^{2} y}{y^{r-1}}-\frac{\ln ^{2}(y+1)}{(y+1)^{r-1}}>0 .
$$

Therefore $\frac{\partial f}{\partial r}(y, r)$ is monotonically increasing with respect to $r$, which means

$$
\sup _{1 \leq \xi \leq r}\left|\frac{\partial f}{\partial r}(y, \xi)\right|=\left|\frac{\partial f}{\partial r}(y, r)\right|=\left|\frac{-\ln y}{y^{r-1}}+\frac{\ln (y+1)}{(y+1)^{r-1}}\right| \leq 2 \frac{-\ln y}{y^{r-1}} .
$$

This completes the proof of the lemma.

Finally we prove Lemma 3.2. By using the previous lemma and integration by parts,

$$
\begin{aligned}
& \int_{-1}^{0} \int_{0}^{\varepsilon} \frac{y^{r}}{(y-x)^{r}} d y d x=\int_{0}^{\varepsilon} y^{r} \int_{-1}^{0} \frac{1}{(y-x)^{r}} d x d y \\
& \quad=\int_{0}^{\varepsilon} y^{r} \frac{y^{1-r}-(y+1)^{1-r}}{r-1} d y \leq \int_{0}^{\varepsilon}-2 y \ln y d y \\
& \quad=\varepsilon^{2}(-\ln \varepsilon+1 / 2) \leq-2 \varepsilon^{2} \ln \varepsilon .
\end{aligned}
$$

By setting $\tilde{y}=\varepsilon-y$ and $\tilde{x}=\varepsilon-x$, one has

$$
\begin{gathered}
\int_{0}^{\varepsilon} \int_{\varepsilon}^{1} \frac{(\varepsilon-y)^{r}}{(x-y)^{r}} d x d y \leq \int_{0}^{\varepsilon} \int_{\varepsilon}^{1+\varepsilon} \frac{(\varepsilon-y)^{r}}{(x-y)^{r}} d x d y \\
=\int_{-1}^{0} \int_{0}^{\varepsilon} \frac{\tilde{y}^{r}}{(\tilde{y}-\tilde{x})^{r}} d \tilde{y} d \tilde{x} \leq-2 \varepsilon^{2} \ln \varepsilon .
\end{gathered}
$$

Since $\frac{3}{2 \ln 2}+1<4$, by using Lemma B.1,

$$
\int_{-1}^{0} \int_{0}^{\varepsilon} \frac{1}{(y-x)^{r}} d y d x=\frac{\varepsilon^{2-r}-(\varepsilon+1)^{2-r}+1}{(r-1)(2-r)} \leq \frac{-4 \varepsilon^{2-r} \ln \varepsilon}{2-r} .
$$


Again, the inequality concerning $\int_{0}^{\varepsilon} \int_{\varepsilon}^{1} \frac{1}{(x-y)^{r}} d x d y$ can be proved by a simple change of variables.

Finally, a simple calculation shows that

$$
\int_{-1}^{0} \int_{0}^{\varepsilon} \frac{y^{2}}{(y-x)^{2}} d y d x=\varepsilon-\ln (\varepsilon+1) \leq \varepsilon^{2} / 2 .
$$

The inequality involving $\int_{0}^{\varepsilon} \int_{\varepsilon}^{1} \frac{(\varepsilon-y)^{2}}{(x-y)^{2}} d x d y$ can be proved similarly.

\section{REFERENCES}

[1] C. Amrouche and V. Girault, On the existence and regularity of the solution of Stokes problem in arbitrary dimension, Proc. Japan Acad. 67 (1991), 171-175. MR1114965 (92i:35098)

[2] Douglas N. Arnold and Ragnar Winther, Mixed finite element for elasticity, Numer. Math. 92 (2002), 401-419. MR1930384 (2003i:65103)

[3] S.C. Brenner and L.R. Scott, The mathematical theory of finite element methods, SpringerVerlag, Berlin, Heidelberg, 1994. MR.1278258 (95f:65001)

[4] Franco Brezzi and Michel Fortin, Mixed and hybrid finite element methods, Springer-Verlag, New York, 1991. MR1115205 (92d:65187)

[5] Z. Cai and G. Starke, First-order system least squares for the stress-displacement formulation: Linear elasticity, SIAM J. Numer. Anal. 41 (2003), 715-730. MR2004196|(2005e:65180)

[6] Z. Cai, C. Tong, P.S. Vassilevski, and C. Wang, Mixed finite element methods for incompressible flow: Stationary Stokes equations, Preprint.

[7] Z. Cai and Y. Wang, A multigrid method for the pseudostress formulation of Stokes problems, SIAM J. Sci. Comp. 29 (2007), 2078-2095. MR2350022

[8] L. Cattabriga, Su un problema al contorno relativo al sistema di equazioni di Stokes, Rend. Sem. Univ. Padova 31 (1961), 308-340. MR0138894 (25:2334)

[9] Philippe G. Ciarlet, The finite element method for elliptic problems, North-Holland, Amsterdam, 1978. MR0520174 (58:25001)

[10] M. Crouzeix and P.A. Raviart, Conforming and nonconforming finite element methods for solving the stationary Stokes equations, R.A.I.R.O. R3 (1973), 33-76. MR0343661 (49:8401)

[11] H. Elman, D. Silvester, and A. Wathen, Finite elements and fast iterative solvers: With applications in incompressible fluid dynamics, Oxford University Press, New York, 2005. MR.2155549 (2006f:65002)

[12] R.S. Falk and J.E. Osborn, Error estimates for mixed methods, RAIRO. Numer. Anal. 14 (1980), 249-277. MR592753 (82j:65076)

[13] G.J. Fix and M.D. Gunzburger, On finite element approximations of problems having inhomogeneous essential boundary conditions, Comp. Math. Appl. 9 (1983), 687-700. MR726817 (85b:65102)

[14] G.P. Galdi, C.G. Simader, and H. Sohr, On the Stokes problem in Lipschitz domains, Annali di Matematica pura ed applicata CLXVII (1994), 147-163. MR.1313554 (95m:35142)

[15] Vivette Girault and Pierre Arnaud Raviart, Finite element methods for Navier-Stokes equations, Springer Series in Computational Mathematics, no. 5, Springer-Verlag, New York, 1986. MR851383 (88b:65129)

[16] P. Grisvard, Singularités des solutions du problème de Stokes dans un polygone, Univ. de Nice., 1979.

[17] _ Elliptic problems in nonsmooth domains, Pitman, Boston, 1985. MR775683 (86m:35044)

[18] M.D. Gunzburger and S.L. Hou, Treating inhomogeneous essential boundary conditions in finite element methods and the calculation of boundary stresses, SIAM J. Numer. Anal. 29 (1992), 390-424. MR1154272 (93d:76039)

[19] P. Hood and C. Taylor, A numerical solution of the Navier-Stokes equations using the finite element technique, Comp. and Fluids 1 (1973), 73-100. MR0339677 (49:4435)

[20] D.D. Joseph and L. Sturges, The convergence of biorthogonal series for biharmonic and Stokes flow edge problems. II, SIAM J. Appl. Math. 34 (1978), 7-27. MR0475146 (57:14765)

[21] H.K. Moffatt, Viscous and resistive eddies near a sharp corner, J. Fluid Mech. 18 (1964), $1-18$. 
[22] P.A. Raviart and J.M. Thomas, A mixed finite element method for second order elliptic problems, Mathematical aspects of the finite element method (I. Galligani and E. Magenes, eds.), Lecture notes in Mathematics, Vol. 606, Springer-Verlag, 1977. MR0483555 (58:3547)

[23] P.N. Shankar, The eddy structure in Stokes flows in a cavity, J. Fluid Mech. 250 (1993), 371-383.

Department of Mathematics, Purdue University, West Lafayette, Indiana 47907

Department of Mathematics, Oklahoma State University, Stillwater, Oklahoma 74078 